Simeon Thompson

\title{
Beethoven und der Zweite Weltkrieg in der künstlerischen \\ Reflexion der Nachkriegszeit. Stanley Kubricks A Clockwork \\ Orange und Rolf Liebermanns Leonore 40/45
}

Die Problematik von Beethoven und der Rolle, die seiner Musik durch ihre politische Instrumentalisierung in der Zeit des Nationalsozialismus zukam, wurde in der Nachkriegszeit vielfach reflektiert, noch öfter aber verdrängt. Solche Reaktionen lassen sich aber nicht nur in musik- und anderem geisteswissenschaftlichem Rahmen, sondern auch in künstlerischen Referenzen auf den Komponisten beobachten. In diesem Aufsatz werden zwei sehr unterschiedliche Beispiele besprochen: Der für seine Beethoven-Rezeption notorische Film A Clockwork Orange von Stanley Kubrick sowie die Oper Leonore 40/45 des Schweizer Komponisten Rolf Liebermann.

Stanley Kubricks Verfilmung von Anthony Burgess' I962 erschienenem Roman A Clockwork Orange gelangte 1972 in die Kinos. Sowohl in der Vorlage als auch in der Adaption kommt dem Bezug zu Beethoven große Bedeutung zu. ${ }^{\mathrm{I}}$ Alex, der Protagonist dieser Geschichte, die in einer dystopischen, nahen Zukunft angesiedelt ist, hält sich für einen besonders kultivierten Liebhaber von Beethovens Musik - nur wird der Komponist immer wieder zum Trieb in Bezug gesetzt, Trieben, denen Alex mehr oder weniger ungehemmt folgt: zu Gewalt, Sex und vor allem zu sexueller Gewalt. Kubrick setzt Beethoven und neben ihm die Werke anderer klassischer Komponisten gezielt als Filmmusik ein, wobei insbesondere Beethoven immer wieder auf der Ebene der Handlung vorkommt. An mehreren Stellen spielt er sogar eine Schlüsselrolle. Verschiedene der Eskapaden und Verbrechen von Alex werden mit Beethovens Musik unterlegt, zum Teil sogar durch sie ausgelöst. Nachdem die Polizei Alex fasst, wird er einer experimentellen Therapie unterzogen, die seine Triebe unterdrücken soll. Durch das »Ludovico Treatment « (wie die Therapie auf Beethoven anspielend heißt) wird Alex ein buchstäblich gefesselter

1 Vgl. David J. Code: Don Juan in Nadsat. Kubrick's Music for A Clockwork Orange, in: Journal of the Ropal Musical Association 139/2 (2014), S.339-386; Peter Höyng: Ambiguities of Violence in Beethoven's Ninth through the Eyes of Stanley Kubrick's A Clockwork Orange, in: The German Quarterly 84/2 (20II), S. I59-I76; Kate McQuiston: Value, Violence, and Music Recognized. A Clockwork Orange as Musicology, in: Stanlep Kubrick. Essaps on His Films and Legacp, hg. von Gary D. Rhodes, Jefferson 2008, S. I05-I22; Peter J. Rabinowitz: A Bird of Like Rarest Spun Heavenmetal. Music in A Clockwork Orange, in: Stanley Kubrick's A Clockwork Orange, hg. von Stuart Y. McDougal, Cambridge 2003 (Cambridge Film Handbooks), S. I09-130; Galia Hanoch-Roe: Beethoven's Ninth. An »Ode to Choice« as Presented in Stanley Kubrick's A Clockwork Orange, in: International Review of the Aesthetics and Sociology of Music 33/2 (2002), S. I7I-I79. 
Betrachter von gewaltvollen Filmsequenzen: Vorab unter Drogen gesetzt, erwachsen Alex negative Assoziationen zu seinen gewaltvollen Trieben. Dabei entwickelt er aber auch unfreiwillig eine Aversion gegen Beethoven, nachdem dessen Musik - genauer die Neunte Sinfonie - während einer der Filmsequenzen erklingt.

Die selbstreflexiven Spiele drängen sich regelrecht auf: Während die Zuschauenden von A Clockwork Orange in der ersten Stunde des Films mitansehen mussten, wie Beethovens Musik bei allerlei Gewalt- und Sexszenen eingesetzt wurde, so muss plötzlich Alex seinen geliebten Komponisten ebenfalls in einem ,falschen Kontext hören. Und Alex' empörte Reaktion darauf könnte direkt aus dem Munde des Publikums von A Clockwork Orange stammen: »It's a sin! Using Ludwig van like that. He did no harm to anyone, Beethoven just wrote music. $\varkappa^{2}$ Im weiteren Verlauf des Films spitzt sich die Verbindung nochmals zu, als sich ein ehemaliges Opfer von Alex an diesem rächt, und zwar mittels akustischer Folter: Übergroße Lautsprecher beschallen Alex mit Beethovens Neunter, die der Protagonist seit der Therapie nicht mehr erträgt - in der Folge stürzt er sich aus dem Fenster des Dachstocks, in dem er eingeschlossen ist. Als sich danach eine politische Partei seiner Sache annimmt und seine nun als inhuman bezichtigte Therapie rückgängig macht, ist auch Alex' Verhältnis zu Beethoven >geheilt : In der letzten Sequenz fantasiert er eine Gewaltorgie, wieder mit Begleitung der Neunten.

A Clockwork Orange - sowohl die Vorlage als auch der Film - will offensichtlich als provozierende Stellungnahme zur hier auf die Spitze getriebenen Spannung zwischen Trieben und Zivilisierung verstanden werden. Die Auseinandersetzung mit Beethoven ist ebenfalls etwas gar dick aufgetragen, wirft aber vor allem Fragen auf, ohne einfache Antworten zu liefern. Was sollte hier über Beethoven gesagt beziehungsweise mit ihm gezeigt werden? Seine Musik, vielleicht auch die Musik überhaupt, steht offensichtlich in einer engen Verbindung mit dem Trieb. Das legt nicht nur die vielfache Verknüpfung auf Ebene der Filmmusik wie der Handlung nahe, sondern auch das Resultat der Therapie: Alex ist danach zwar gut angepasster Bürger und erträgt nicht einmal den Gedanken an Gewalt, aber er erträgt auch Beethoven nicht mehr.

Die damit nahegelegte Bedeutung von Beethoven gestaltet sich jedoch vielschichtiger. Gerade der Einsatz in Alex' Therapie war von Ärzten im Film nicht geplant: Die Musik ist ihnen zufolge lediglich der \Soundtrack $<$ des betreffenden Filmausschnitts, der Alex gezeigt wird. Doch handelt es sich dabei nicht um eine weitere Gewaltszene, sondern allem Anschein nach um ein historisches Dokument, und zwar nationalsozialistische Propaganda. ${ }^{3}$ In Kubricks Drehbuch waren für diese Stelle sogar Aufnahmen aus einem

3 Der an sich naheliegende Schluss, es handle sich bei der Beethoven-Unterlegung um eine authentische Gegebenheit - dass die nationalsozialistische Propaganda also bereits im Original mit Beethoven 
Konzentrationslager vorgesehen gewesen. Durch die Änderung wird die hier nahegelegte Assoziation mit Beethoven spezifiziert: Der Komponist wird nicht etwa in einem allgemeineren Sinne mit menschlichen Abgründen in Verbindung gebracht, sondern mit dem >Dritten Reichく, und zwar nicht mit den Opfern des Holocaust, sondern mit den Tätern. (Das damit angesprochene Problem, die vielfach beschworene Verbindung von >Barbarei< und `Kultur`im Nationalsozialismus, war für Kubrick kein bloßes Abstraktum, war er doch über seine Frau nahe mit dem deutschen Regisseur Veit Harlan verwandt, seinerseits verantwortlich für den Propagandafilm Jud Süß.)

»He did no harm to anyone, Beethoven just wrote music«: So lautet die paradigmatische Antwort eines Musikliebhabers, der in seinem Hörerlebnis möglichst ungestört bleiben will von einem lästigen Kontext. Diese Haltung bestimmt auch die Perspektive der Geisteswissenschaft und insbesondere der Musikwissenschaft, wenn sie kanonische Musik und deren >Vereinnahmung durch den Nationalsozialismus in eine Beziehung setzt: Hier die passive Musik und ihre unschuldigen Schöpfer und Schöpferinnen, dort die böswilligen Instanzen, die sie zu missbrauchen trachten. Ein Beethoven könne schließlich nichts dafür, wenn seine Werke hundert Jahre nach seinem Tod in ganz anderen Kontexten erklingen als in denen, für die sie geschaffen wurden.

Die Verwendung, ja gar Inszenierung von Beethoven in A Clockwork Orange lässt an einer solchen Perspektive gehörig Zweifel aufkommen. Ohne in Alex gleich das Abbild eines Nazis zu sehen, weil er kultiviertes, diszipliniertes Auftreten mit nackter Gewalt kombiniert: Der Protagonist selbst wie auch der Film, sie ordnen beide Beethoven entschieden der Welt der Gewalt zu. Eine etwaige Kausalität daraus abzuleiten, bleibt dem Publikum überlassen. Löst Beethoven Gewalt aus, eignet er sich nur als Soundtrack dazu, ist seine Musik sogar aus Gewalt geboren, oder soll damit vielleicht doch ein positiver Ansatz angedeutet werden, etwa eine Bändigung von Trieben in eine zivilisierte Struktur? Eines scheint jedenfalls nach der Logik des Films sicher: Der Komponist lässt sich nicht ohne Weiteres aus den Kontexten lösen, in denen er stand oder in die er gestellt wurde, und dazu gehört auch das zentrale Trauma des 2o. Jahrhunderts, der Holocaust.

Demgegenüber muss ein Werk wie Leonore 40/45 in seiner Verharmlosung geradezu verheerend wirken. Die Oper wurde vom Schweizer Komponisten Rolf Liebermann auf unterlegt war, bevor das Filmmaterial für Alex' Therapie verwendet wurde -, wird dadurch problematisiert, dass die Neunte hier in einer verfremdeten, elektronisch erzeugten Interpretation von Wendy Carlos erklingt. Der für I972 doch recht futuristisch anmutende Klangeindruck suggeriert, dass diese Beethoven-Interpretation zeitlich in der von A Clockwork Orange dargestellten Zukunft anzusiedeln ist. 
ein Libretto komponiert, das der deutsche Musikwissenschaftler Heinrich Strobel verfasst hatte; das Werk wurde 1952 in Basel uraufgeführt und erlebte danach auch Aufführungen in Deutschland. ${ }^{4}$ Die Oper bemüht sich mittels einer allegorischen Handlung, die Wunden des Zweiten Weltkriegs zu heilen. Alfred, ein nur widerwillig kämpfender deutscher Soldat, lernt während der Besatzung von Paris Huguette kennen und lieben. Nach dem Krieg ist Alfred zwar zunächst interniert, aber Huguette findet ihn bald, sodass die beiden mitsamt ihren Familien Hochzeit feiern können. Die Allegorie wird durchgängig von der Symmetrie der deutschen und französischen Familien bestärkt, sodass die Hochzeit schließlich nichts weniger darstellen soll als das friedliche Ende des Konflikts - eines Konflikts, der natürlich alles andere als gelöst war, weder in der Handlungszeit unmittelbar nach Kriegsende noch sieben Jahre später, als die Oper uraufgeführt wurde.

Dieser beinahe utopisch anmutende Optimismus wäre an sich kaum ernst zu nehmen. Aber die Autoren statteten ihre Oper mit einer gehörigen Portion Ironie und Selbstreflexivität aus und verliehen dem Werk somit einen frechen, entschieden >modernen $<$ Anstrich. Im Dialog mit dem Schutzengel Monsieur Emile - dieser sorgt dafür, dass sich alles zum Guten wendet - führt die Protagonistin Huguette explizit aus, wie ihre Vereinigung mit Alfred ihren Völkern ein »Beispiel« sein soll:

»Huguette: Es muß endlich Schluß sein mit dem Haß zwischen unseren Völkern - und Schluß mit den Kriegen. Was Alfred und mir gelang, muß auch im Großen gelingen. Die Liebe ist stärker als der Krieg; wir wollen ein Beispiel sein [...].

Monsieur Emile [...]: Glaubt Ihr, daß Eure sentimentalen Affären den Lauf der Geschichte verändern werden?

Huguette: Ja, Monsieur Emile, man muß einen Anfang machen. Die andern sollen sehen, daß es so etwas gibt, und daß es hält, und daß die Liebe mehr Gewalt hat als tausend Jahre >Erbfeindschaft««.5

Huguettes Optimismus lässt sich kaum beim Wort nehmen, vor allem nachdem sich Monsieur Emile mit seinem eröffnenden Monolog direkt ans Publikum gewandt hatte:

»ich bin's - der rettende Engel - [...] ein Phantasiegebild, das man, ach! auf den tönenden Brettern braucht - und sogar in dieser höchst banalen Geschichte, die man Ihnen vorführen wird - eine Liebesgeschichte natürlich - sonst wären wir ja nicht in der Oper ...

Aber eine Liebesgeschichte aus der Gegenwart. Sie runzeln die reizende Stirn, meine Schönen? ${ }^{6}$

4 Zu Leonore vgl. Michael Custodis/Friedrich Geiger: Netzwerke der Entnazifizierung. Kontinuitäten im deutschen Musikleben am Beispiel von Werner Egk, Hilde und Heinrich Strobel, Münster 2013 (Münsteraner Schriften zur zeitgenössischen Musik, Bd. I), S. I58-I62.

5 Heinrich Strobel: Leonore 40/45. Opera semiseria in einem Vorspiel und sieben Bildern (zwei Akte) [Libretto], Wien I952, S.30.

6 Ebd., S.5. 
Mit seiner Haltung - Monsieur Emile ist laut den Bühnenanweisungen "gelangweilt und spricht lässig-arrogant « 7 - und seiner eingeschworenen Kommunikation mit dem Publikum steht er gewissermaßen über dem Geschehen und lädt ab Beginn ein, der »banal « genannten Handlung nicht zu trauen. Die hier demonstrierte Skepsis nimmt zugleich aber jede Kritik gegen die Glaubwürdigkeit der Handlung vorweg.

Am Ende darf die »Liebesgeschichte aus der Gegenwart« ohne bissigen Kommentar ihren Lauf nehmen. Der vormals sarkastisch auftretende Monsieur Emile leitet zum Schluss einen sechsstimmigen Chor ein mit dem Text: »Alles wendet sich zum Guten in der besten aller Welten.$^{8}$ Der Schutzengel paraphrasiert hier zwar Leibnitz, aber für das Publikum ist die Anspielung auf Dr. Pangloss, den unverbesserlichen Optimisten in Voltaires Candide, mehr als offensichtlich. Was zunächst wie eine ironische Spitze anmutet, erschöpft sich jedoch im ironischen Gestus oder muss ganz und gar unironisch verstanden werden: Anders als in Candide stellt die Handlung in Leonore 40/45 die These von der besten aller möglichen Welten nie in Frage - geschweige denn, dass die Handlung aus einer Reihe von Gegenbeweisen bestehen würde, wie dies bei Voltaire der Fall ist.

Die Oper trägt eine sehr optimistische, hoffnungsvolle Botschaft, an der ein gesunder Zweifel nagt - aber nicht genug, dass sie sich hinterfragen ließe. Auch die Selbstreflexion, die die Autoren ihrer als solche gekennzeichneten Allegorie eingeschrieben haben, wirkt eher demonstrativ, als dass sich Liebermann und Strobel damit an ihrem Medium oder an einer Tradition abarbeiten wollten. Die Allegorie will doch instruktiv verstanden werden, eben den Menschen ein »Beispiel« sein; dass die Oper mit ein wenig Selbstreflexion und Ironie gepfeffert ist, soll sie einem vielleicht skeptischen Publikum etwas verdaulicher machen.

Ein wichtiges selbstreferentielles Element nimmt gleich der Titel vorweg: Es handelt sich um die zahlreichen Bezüge zu Beethoven und insbesondere zu Fidelio. Hier wie da befreit eine Protagonistin ihr männliches Pendant; zudem fallen mehrere strukturelle und musikalische Anspielungen ins Auge, etwa die an Fidelio angelehnten Ensembles. Zum Beginn der Oper lauscht sogar eine Figur Beethovens Oper über den Rundfunk. Liebermann und Strobel arbeiten sich aber so wenig an Fidelio ab wie an der Allegorie: Auch hier reicht die Selbstreflexion nicht weit über ein wissendes Zwinkern hinaus.

Selbst wenn man dem Werk einen tieferreichenden Zwiespalt zugesteht, bleibt es suggestiv. Die Ironie bietet einem Publikum an, der Sache zu trauen oder nicht. Die hier dargestellte heile Welt mag also glaubwürdig oder illusorisch sein. Analog dazu stünde ein Werk wie Fidelio als zeitloser Beweis für das Gute im Menschen oder eben nicht, und

7 Ebd.

8 Ebd., S. 43 . 
Beethoven wäre mit seinem Werk genauso realitätsfern wie Dr. Pangloss in Candide. Ein solches Interpretationsangebot ließe sich auch auf einer übergeordneten Ebene in Bezug auf die Beethoven-Interpretation im Allgemeinen weiterdenken: Beethoven und seine Oper hätten entweder der Epoche nach dem Krieg etwas zu sagen - genauer: es ließe sich mit ihnen etwas sagen - oder aber Beethoven wäre ganz Kind seiner Zeit, das heißt einer etwas gar optimistischen Aufklärung. Sofern die Selbstreflexion in Leonore 40/45 überhaupt für eine solche Ambiguität tragfähig ist, bestimmt das Werk im selben Schritt die Parameter dieser Ambiguität und schließt damit einen weit schwierigeren Ansatz kategorisch aus: die Möglichkeit nämlich, dass Beethoven weder ein positiver Bezugspunkt noch irrelevant ist, sondern ein negativer Bezugspunkt-Teil des Bösen, Teil der Gewalt, gegen die sich die Oper eigentlich aussprechen will.

Gleich zu Beginn des Werks findet sich eine Stelle, die sich in diesem Sinne gegen den Strich lesen lässt. Der Vater von Alfred (er trägt den `deutschen Namen par excellence, Hermann) hört die bereits genannte Aufführung von Fidelio im Rundfunk, doch bevor sein geliebtes Quartett erklingen kann, wird die Übertragung unterbrochen durch eine Sondermeldung, die alle wehrpflichtigen Männer einberuft. 9 Die intendierte Symbolik ist klar genug: Die Welt des Krieges und des Nationalsozialismus steht in einem schroffen Gegensatz zur Welt von Beethoven und Fidelio, die Nazis müssen Beethoven abklemmen, um den Krieg auszurufen.

Man braucht aber kein Spezialwissen - und man brauchte es schon gar nicht I952, als die Oper uraufgeführt wurde -, um erstens zu wissen, dass der Rundfunk ein wichtiges Medium für nationalsozialistische Propaganda war, und zweitens, dass mit Beethovens Musik und unter Berufung auf sie Krieg gemacht wurde. Es war und ist genug bekannt, dass sich Vertreter des Nationalsozialismus gerne auf das Erbe der >großen $<$ deutschen Musik beriefen, sie in einen unmittelbaren Zusammenhang mit dem Nationalsozialismus stellten und den Überlegenheitsanspruch der deutschen Kultur als wichtigen Bestandteil ihrer Politik nutzten. Beispiele für ihre Beethoven-Interpretation sind zahlreich. ${ }^{\text {IO }}$ Die Verwendung von Fidelio zu Beginn von Leonore 40/45 inszeniert einen Bruch, doch weist die Übertragung von Beethoven im deutschen Rundfunk im Sommer I939 auf eine Kontinuität hin. Eine Aufführung oder Rundfunkübertragung von Beethovens Musik stand nicht quer zum Nationalsozialismus, nicht einmal zur Kriegsführung, sondern war darin integriert, also letztlich Teil davon.

Liebermann und Strobel bemühen sich fast krampfhaft darum, die Last des Nationalsozialismus zu überwinden, indem sie ihn gar nicht erst thematisieren. Über Politik 
hört man wenig, nur dass Alfreds Vater Hermann ein »alter Sozi «II sei und somit mit den Nazis und allgemein mit dem Krieg nichts am Hut habe. Darin sind sich ohnehin alle Figuren einig, Alfred dient beispielsweise in der Militärmusik, feuert somit »nicht einen Schuß «ab, wie Huguette später berichtet. ${ }^{\text {I2 }}$ Überhaupt kommen in der Oper - dies im markanten Gegensatz zu Fidelio - keine negativen Figuren vor. Ohne von Liebermann und Strobel erwarten zu wollen, dass sie sich in Leonore 40/45 grundlegend mit dem Problem des Nationalsozialismus befassen: Selbst das spezifische, gut bekannte und hier brisante Problem der nationalsozialistischen Aneignung von Beethoven wird nicht in Angriff genommen, sondern gänzlich ausgeklammert.

In ihrem Bemühen, um jeden Preis eine möglichst friedliche Perspektive für das kriegszerrissene Europa aufzuzeigen, ist die Oper typisch für ihre Zeit und besonders für die Schweiz jener Jahre. Hier entstanden unmittelbar vor und nach Kriegsende eine Reihe von heute eher befremdlich anmutenden Kunstwerken mit vergleichbarer Stoßrichtung. Bereits vor Kriegsende erhielt beispielsweise Frank Martin den Auftrag, für Radio Genève ein Werk zu komponieren, das bei Kriegsende erklingen sollte. Er vertonte für In terra pax diverse Bibeltexte und fügte sich somit auch einem vorherrschenden nationalen Sendungsbewusstsein: Die Schweiz verstand sich als neutrales Land, das dazu berufen war, zwischen den zerstrittenen Ländern zu vermitteln und für den Frieden einzustehen ${ }^{\mathrm{I} 3}$ - eine Rolle, die mit solchen religiös anmutenden Werken (Honeggers Spmphonie liturgique gehört ebenfalls dazu) sozusagen ins Schicksalhafte, Heilige überhöht wurde. Dieses Selbstverständnis findet sich im Übrigen auch in Leonore 40/45 ganz direkt wieder: Wie das fünfte Bild zeigt, ist der Schutzengel weder in Deutschland noch in Frankreich, aber auch nicht im Himmel zuhause, sondern in den idyllischen Schweizer Alpen, in einem »Châlet am Blumenpfad « »[z]wischen Chur und Wallenstadt $\ll^{\mathrm{I}}{ }^{4}$

Max Frisch thematisierte zur selben Zeit in Nun singen sie wieder (1945) und Als der Krieg zu Ende war (I948) mit gleich zwei Werken die Schäden des Kriegs, wobei besonders im ersteren Werk - nicht unähnlich zu Liebermann und Strobel - dem Krieg eine elementare Dimension zukommt und eine Äquivalenz zwischen beiden Seiten beziehungsweise eine beidseitige Schuld nahegelegt wird. (Im Übrigen irritiert das wohl bekannteste Werk, das im weitesten Sinne dem zeitgenössischen Schweizer Kontext zuzuordnen ist,

Strobel: Leonore 40/45, S. 8 .

Ebd., S. 29.

Vgl. Jakob Tanner: Geschichte der Schweiz im 20. Jahrhundert, München 2015 (Europäische Geschichte im 20. Jahrhundert), S. 292-297.

Strobel: Leonore 40/45, S. 27, gleichzeitig eine Anspielung an Igor Strawinskys Histoire du soldat, die in Hans Reinharts Übersetzung mit eben diesen Worten beginnt. Dort bedingt der Rhythmus das Doppel-l im Städtenamen. Ganz herzlichen Dank an Thomas Gartmann für den Hinweis. 
aus ähnlichen Gründen: Brechts I94I in Zürich uraufgeführte Mutter Courage und ihre Kinder.)

Immerhin geht Frisch aber auf den Zusammenhang zwischen kanonischer deutscher Kultur, insbesondere Musik, und dem Krieg ein. In Als der Krieg zu Ende war etwa erscheint die beanspruchte Unschuld eines deutschen Pianisten, der sich auf Mozart beruft, in einem mehr als zweifelhaften Licht. ${ }^{\mathrm{I5}}$ Wenn Liebermann und Strobel dieser Thematik gänzlich auswichen, so scheinen die Autoren also selbst für ihren unmittelbaren Schweizer Kontext besonders zurückhaltend gewesen zu sein. Dies, obwohl sich bei Leonore 40/45 die Frage nach der Beethoven-Rezeption im Krieg fast unweigerlich stellt und die Autoren keine Hemmungen zeigten, die nationalsozialistische Kulturpolitik stellenweise darzustellen, wenn es etwa um die Legitimierung der Zwölftontechnik ging, die ein Deutscher Zuhörer während eines Pariser Konzerts empört kommentiert: »So ein Unfug -, wäre bei uns längst verboten. « ${ }^{\mathrm{I}}$

Die Beethoven-Rezeption zur Zeit des Nationalsozialismus bildet in der Oper sozusagen einen >Elephant in the room<, über den sich die Autoren ausschweigen. Ohne über Heinrich Strobels Beweggründe zu spekulieren ${ }^{\mathrm{I} 7}$ lässt sich diese Zurückhaltung mit der Situation von Rolf Liebermann erklären. In der Schweiz hatte das Verständnis der Neutralität während der Ns-Zeit zum Teil groteske Konturen angenommen, wie sich etwa in Auseinandersetzungen zu literarisch tätigen Exilanten und Exilantinnen beobachten lässt: Jüdischen Kunstschaffenden wurde per se eine neutrale Perspektive abgestritten; der Nationalsozialismus und seine Opfer standen aus Schweizer Sicht in einer Art Gleichgewicht. ${ }^{18}$ Diese Sicht wie auch ein allgemein anhaltender Antisemitismus präg-

15 "Was hat unsereiner verbrochen? Ich bitte Sie. Nur weil meine großen Erfolge gerade in diese Zeit fielen. Was hat Mozart zu tun mit dem Dritten Reich? Und was habe ich anderes getan: als Mozart gespielt - mitten im Luftterror, ich bitte Sie, Frühjahr neunzehndreiundvierzig!« Max Frisch: Santa Cruz, Nun singen sie wieder, Die chinesische Mauer, Als der Krieg zu Ende war, Kleine Prosaschriften, Tagebuch I946-I949, Frankfurt a. M. 1998 (Gesammelte Werke in zeitlicher Folge, Bd. 2), S. 266.

16 Strobel: Leonore 40/45, S. I2.

17 Zu Strobel und dessen Aktivitäten in der Nachkriegszeit vgl. Custodis/Geiger: Netzwerke der Entnazifizierung.

18 Vgl. zu Kultur und Politik in der Schweiz während der Ns-Zeit etwa Kristina Schulz: Die Schweiz und die literarischen Flüchtlinge (I933-I945), Berlin 2012 (Deutsche Literatur. Studien und Quellen, Bd.9); Ursula Amrein: »Los von Berlin!«. Die Literatur- und Theaterpolitik der Schweiz und das »Dritte Reich«, Zürich 2004; Hans Ulrich Jost: Bedrohung und Enge (I9I4-I945), in: Geschichte der Schweiz und der Schweizer, hg. von Beatrix Mesmer, Basel 2006, S. 73I-8I9; Philipp Sarasin: Metaphern der Ambivalenz. Philipp Etters »Reden an das Schweizervolk« von I939 und die Politik der Schweiz im Zweiten Weltkrieg, in: ders.: Geschichtswissenschaft und Diskursanalyse, Frankfurt a. M. 2003, S. I77-I90; Matthias Kassel: »Begründung: Überfremdung - die Schweiz als Exilland während des Zweiten Weltkriegs, in: »Entre Denges et Denezy ...«. Dokumente zur Schweizer Musikgeschichte I900-2000, hg. von dems. und Ulrich Mosch, Mainz u.a. 2000, S.365-375; Josef Mooser: Die »Geistige Landesverteidi- 
ten die frühe Nachkriegszeit. In diesem Kontext hätte ein jüdischer Künstler wie Liebermann mit viel mehr Kritik zu rechnen gehabt als ein Max Frisch, über dessen Neutralität keine vergleichbaren Zweifel bestanden.

Leonore 40/45 und A Clockwork Orange betten Beethoven in allegorische, wenn nicht sogar platte Handlungen ein, aber jeweils auf sehr selbstreflexive Art und Weise. Kubrick und Liebermann, beides Künstler mit jüdischem Hintergrund, stellen dabei grundlegend verschiedene Interpretationen von Beethoven zur Diskussion: Liebermann macht einen möglichst großen Bogen um die Kontextualisierung des Komponisten im Nationalsozialismus, während Kubrick direkt auf diesen wunden Punkt zusteuert. Dieser Unterschied ist in erster Linie auf die ganz verschiedenen Entstehungskontexte der Werke zurückzuführen. In der Schweiz der Nachkriegszeit brauchte Liebermann ausgesprochen viel Mut, um überhaupt die Thematik des Krieges aufzugreifen, wenn auch mit einem sehr versöhnlichen Ansatz.

Der Unterschied zu Kubrick erklärt sich aber wohl auch durch eine grundlegend andere Perspektive auf Beethoven. Liebermann mag tatsächlich gehofft haben, mit seiner Oper zu einer Aussöhnung beizutragen. Nur wenige Jahre nach Kriegsende, in einer Zeit, in der die Wunden alles andere als geheilt waren, scheint der Zweifel eines Monsieur Emile zwar glaubwürdiger als die Zuversicht der Huguette, unabhängig davon, wer das letzte Wort hat. Liebermann und wohl auch einem großen Teil seines Publikums wird bewusst gewesen sein, dass eine solche Harmonie vorerst nur »auf den tönenden Brettern« möglich war. Dafür aber, dass Liebermann an der versöhnenden Kraft von Beethovens Musik zweifelte, findet man an keiner Stelle der Oper auch nur das leiseste Anzeichen.

gung « in den I930er Jahren. Profile und Kontexte eines vielschichtigen Phänomens der schweizerischen politischen Kultur in der Zwischenkriegszeit, in: Schweizerische Zeitschrift für Geschichte 47/4 (I997), S. 685-708. 


\section{Inhalt}

Vorwort 8

INTERPRETATION - BEGRIFF, METHODE, PRAXIS

Laure Spaltenstein Interpretation als treue Übersetzung. Zur Frühgeschichte eines vieldeutigen Begriffs I5

Kai Köpp Von der Quelle zur Methode. Zum Entwurf einer historischen Interpretationsforschung $\quad 28$

Manuel Bärtsch >Interpretation‘. Beethovens Sonate A-Dur op. IOI in der Sicht von Eugen d'Albert und Frederic Lamond

Sebastian Bausch Klavierrollen als Interpretationsdokumente. Ein Erfahrungsbericht als Leitfaden für Einsteiger $\quad 7 \mathrm{I}$

Camilla Köhnken Beethoven-Auslegung zwischen Liszts »Deklamationsstil« und Bülows »Vivisektionsversuchen«. Auf den Spuren Liszt'scher Interpretationsideale in Hans von Bülows instruktiver Edition der Klaviersonaten Beethovens $\quad 92$

Neal Peres Da Costa Carl Reinecke's Performance of his Arrangement of the Second Movement from Mozart's Piano Concerto K. 488. Some Thoughts on Style and the Hidden Messages in Musical Notation

II4

Carolina Estrada Bascuñana Enrique Granados's Performance Style.

Visualising the Audible Evidence I5O

Lukas Näf Tempogestaltung in Weberns Sinfonie op. 2I I80

INTERPRETATION - AUFFÜHRUNGSGESCHICHTE

Christoph Moor "Ein so erklärtes Lieblingsstück der hiesigen Kunstfreunde«. Die Rezeptionsgeschichte der Jupiter-Sinfonie in Beethovens Wien

Luisa Klaus Objektive Bruckner-Interpretation? Zur Aufführung der Trio-Entwürfe für die Neunte Sinfonie I940 205

Chris Walton Von innen und von außen. Beethovens Neunte Sinfonie und die $>$ Wagner'sche< Dirigiertradition $\quad 2 \mathrm{I} 8$

Lena-Lisa Wüstendörfer Streit um Fidelio. Gustav Mahler und Felix Weingartner im Disput um Werktreue $\quad 238$

INTERMEZZO

Robert Levin Turning Point to Musical Modernity. Beethoven as Executor of the Legacy of C. P. E. Bach. Concert Lecture 249

INTERPRETATION - INSTRUMENTE, ANALYSE, EDITION

Martin Skamletz «Man hat diese Erweiterung des Tonumfanges seit ein paar Jahren an den Tasteninstrumenten sehr weit getrieben.« Der Umgang mit Grenzen beim späten Mozart und beim frühen Beethoven $\quad 263$ 
Stephan Zirwes Analyse und Interpretation. Adolph Bernhard Marx' Beethoven-Analysen 29I

Michael Ladenburger Was können wir aus Originalhandschriften von Beethoven für eine angemessene Interpretation lernen? 30I

Federica Rovelli Die Skizzenbuch-Ausgaben und ihre mögliche digitale Zukunft 3 I7

Johannes Gebauer Interpretationspraktische Stemmatik. Philologische Methoden in der Interpretationsforschung am Beispiel annotierter Notenausgaben von Rodes 24 Capricen und Beethovens Violinkonzert

John Rink Chopin Copying Chopin 349

Tomasz Herbut Alexander Goldenweiser und Beethovens Sonate op. IIo- eine Spurensuche 366

INTERPRETATION - KREATIVE ANEIGNUNG

Thomas Gartmann Beethoven als sein eigener Interpret. Gedanken zur Bearbeitung der Klaviersonate op. I4/ז für Streichquartett

Ivo Haag Die Sinfonien von Johannes Brahms - (auch) Klaviermusik?

Michael Lehner Das Orchester auf dem Klavier. Welte-Klavierrollen von Gustav Mahler und Richard Strauss als interpretationsanalytische Quellen

Roger Allen “That Is What Music Really Is". Richard Wagner's Reception of Beethoven's Piano Sonata in A Major Op. IOI $43 \mathrm{I}$

Daniel Allenbach Eine heroische< Neunte? Dmitri Schostakowitschs Neunte Sinfonie im Vergleich mit Ludwig van Beethoenvs Sinfonien Nr.3 und 9 44I

Simeon Thompson Beethoven und der Zweite Weltkrieg in der künstlerischen Reflexion der Nachkriegszeit. Stanley Kubricks A Clockwork Orange und Rolf Liebermanns Leonore 40/45 456

Michelle Ziegler Rettungsversuch im Jubiläumsjahr. Mauricio Kagels Aufarbeitung der Beethoven-Rezeption in der Ludwig van-Werkgruppe (I970) 465

Leo Dick Über den späten Beethoven zur >Postidentität،. Die Suche nach liminalen Räumen im gegenwärtigen Musiktheater am Beispiel von Matthias Rebstocks Berliner Produktion Büro für postidentisches Leben $\quad 476$

Elizabeth Waterhouse Choreographic Re-mix. William Forsythe's Trio (I996) and Beethoven's String Quartet No. I5 in a Minor Op. I32 487

László Stachó "Gradus ad Parnassum".

The Purgatory of Instrumental Technique

Namen-, Werk- und Ortsregister 522

Die Autorinnen und Autoren der Beiträge 


\section{Rund um BeEthoven \\ Interpretationsforschung heute • \\ Herausgegeben von Thomas}

Gartmann und Daniel Allenbach 


\section{MUSIKFORSCHUNG DER Hochschule der KÜnste Bern Herausgegeben von Martin Skamletz und Thomas Gartmann Band 14}


0 Dieses Buch ist in gedruckter Form im Dezember 2019 in erster Auflage in der Edition Argus in Schliengen/Markgräflerland erschienen. Gestaltet und gesetzt wurde es im Verlag aus der Seria und der SeriaSans, die von Martin Majoor im Jahre 2000 gezeichnet wurden. Gedruckt wurde es auf Eos, einem holzfreien, säurefreien, chlorfreien und alterungsbeständigen Werkdruckpapier der Papierfabrik Salzer im niederösterreichischen Sankt Pölten. Das Vorsatzpapier Caribic cherry wurde von Igepa in Hamburg geliefert. Rives Tradition, ein Recyclingpapier mit leichter Filznarbung, das für den Bezug des Umschlags verwendet wurde, stellt die Papierfabrik Arjo Wiggins in Issy-les-Moulineaux bei Paris her. Das Kapitalband mit rot-schwarzer Raupe lieferte die Firma Dr. Günther Kast aus Sonthofen im Oberallgäu, die auf technische Gewebe und Spezialfasererzeugnisse spezialisiert ist. Gedruckt und gebunden wurde das Buch von der Firma Bookstation im bayerischen Anzing. Im Internet finden Sie Informationen über das gesamte Verlagsprogramm unter www.editionargus.de, zum Institut Interpretation der Hochschule der Künste Bern unter www.hkb.bfh.ch/interpretation und www.hkb-interpretation.ch. Die Deutsche Nationalbibliothek verzeichnet diese Publikation in der Deutschen Nationalbibliografie; detaillierte bibliografische Daten sind im Internet über www.dnb.de abrufbar. (c) der zeitgleich erschienenen digitalen Version: die Autorinnen und Autoren, 20I9. Dieses Werk ist lizenziert unter einer Creative Commons Namensnennung-Nicht kommerziell 4.0 International Lizenz (CC BY-NC 4.o). DoI: https://doi.org/I0.26045/kp64-6I78 ISBN 978-3-93I264-94-9 